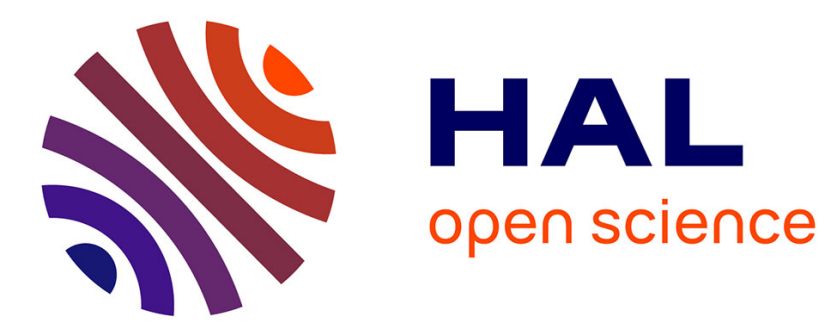

\title{
Non-Reciprocal Scattering in Shear Flow
}

Charlotte Saverna, Yves Aurégan, Vincent Pagneux

\section{To cite this version:}

Charlotte Saverna, Yves Aurégan, Vincent Pagneux. Non-Reciprocal Scattering in Shear Flow. Journal of the Acoustical Society of America, 2019, 146 (2), pp.1051-1060. 10.1121/1.5120523 . hal02336972

\section{HAL Id: hal-02336972 \\ https://hal.science/hal-02336972}

Submitted on 29 Oct 2019

HAL is a multi-disciplinary open access archive for the deposit and dissemination of scientific research documents, whether they are published or not. The documents may come from teaching and research institutions in France or abroad, or from public or private research centers.
L'archive ouverte pluridisciplinaire HAL, est destinée au dépôt et à la diffusion de documents scientifiques de niveau recherche, publiés ou non, émanant des établissements d'enseignement et de recherche français ou étrangers, des laboratoires publics ou privés. 


\title{
Non-Reciprocal Scattering in Shear Flow
}

\author{
Charlotte Saverna, ${ }^{1}$, a) Yves Aurégan, ${ }^{1}$ and Vincent Pagneux ${ }^{1}$ \\ Laboratoire d'Acoustique de l'Université du Mans, Le Mans, 72000, France
}

This work presents a study of scattering phenomena in shear flows and its application to impedance walls. These flows are described by a dimensionless shear layer thickness and a mean Mach number. Both transmission through a given shear layer and reflection on an acoustic treatment are studied. We show that the dimensionless thickness of the shear layer may be an interesting tool to reach perfect absorption or large lateral displacement of a Gaussian beam.

(C)2019 Acoustical Society of America. [http://dx.doi.org(DOI number)]

$[\mathrm{XYZ}]$

\section{INTRODUCTION}

Adding flow to an acoustic system is one of the simplest ways to make it non-reciprocal. Recently, it has been used to obtain sound isolation ${ }^{16}$ or asymmetric propagation $^{39}$, in the broad context of research on unidirectional transmission devices for acoustic waves $^{17,32}$. It appears that sound-flow interactions have been studied for a very long time ${ }^{42}$, early works being motivated by the study of the deflection of sound in the wind ${ }^{12}$. For instance, in the Theory of Sound, Rayleigh ${ }^{45}$ uses geometric acoustics to show in which direction the sound is deflected according to the direction of the wind. Subsequently, varieties of studies were then led on the effect of shear flows on acoustic propagation, from a simple flow velocity discontinuity ${ }^{33,34,47}$, to piece-wise constant profiles $^{1,52}$ or continuous parallel shear flows ${ }^{23,26,28}$. Many other papers deal with the propagation of sound in parallel shear flow in the presence of walls, either for ducts $^{44}$ or for reflection problems ${ }^{5,8,21,25,31,36,40,41}$. For impedance walls, it can be noted that analytic expressions exist for very small boundary layers ${ }^{4,18}$, which tend to the one given by Ingard ${ }^{25}$ and Myers ${ }^{37}$ for vanishing boundary layers.

Though rarely mentioned as such, non-reciprocity is obviously an existing feature in most of the previously cited works. In this paper, we will inspect the effect of basic parallel shear flows on the non-reciprocity properties. In the model we use, both the mean Mach number and the height of the shear layer can be varied independently. Though not so realistic, this will allow us to highlight some key aspects of the influence of shear. We consider two specific scattering problems: i) reflection and transmission, when the fluid is moving at two different velocities above and under a layer of shear flow with a linear profile, ii) reflection, when the fluid is flowing above a lined wall with a shear layer. The first problem we consider is similar to the ones studied in jet-like configurations by Amiet ${ }^{1}$ and Jones $^{26}$, except we consider it as a scattering problem. This enables us to get a uni-

a) charlotte.saverna@univ-lemans.fr
Pages: $1-10$

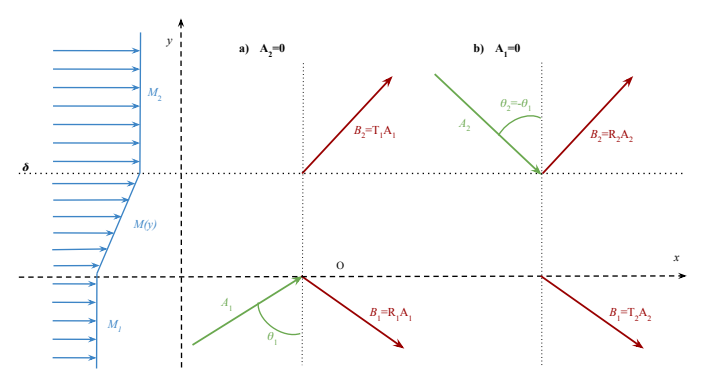

FIG. 1. Scheme of the problem of scattering by a layer of shear flow a) in the case when the incident wave comes from below the shear layer, b) when it comes from above the shear layer.

tary relation on the full scattering matrix. In the second problem, we highlight other non-reciprocal effects such as unilateral perfect absorption or asymmetric lateral displacement of Gaussian beams. The latter phenomenon is known as Goos-Hänchen shift ${ }^{2,22}$ in optics ${ }^{6,10}$ or Schoch effect in ultrasounds ${ }^{13,24,50}$. In the presence of shear flow, we show it is possible to reach large lateral displacement due to non-reciprocity.

\section{GENERAL SCATTERING PROPERTIES OF A SHEAR FLOW}

We consider the problem of the scattering of a plane wave by a layer of shear flow between two fluids moving at constant Mach number $M_{1}$ and $M_{2}$, as described in Fig. 1. We study this problem using a scattering matrix $S$ linking the incident amplitudes $A_{1,2}$ to the scattered amplitudes $B_{1,2}$.

\section{A. Energy conservation}

The propagation equation for a wave in a parallel flow is given by $^{44}$ :

$$
D_{t}\left[\left(\partial_{x}^{2}+\partial_{y}^{2}\right) p-\frac{1}{c_{0}^{2}} D_{t}^{2} p\right]-2 \partial_{y} U(y) \partial_{x, y}^{2} p=0
$$


where $D_{t}$ is a convective derivative term, $D_{t}=\partial_{t}+U \partial_{x}$, and $U(y)$ is the mean velocity of the flow, such that $M(y)=U(y) / c_{0}$, with $c_{0}$ the constant sound speed. To study the energy conservation, non-trivial in shear flow, we consider the pressure $p$ to be a plane harmonic wave, such that $p(x, y, t)=\Re\left[P(y) \mathrm{e}^{-\mathrm{i}(\omega t-\alpha x)}\right]$, where $\omega=2 \pi f$ is the circular frequency, $\alpha=K \sin \left(\theta_{i}\right) /\left(1+M_{i} \sin \left(\theta_{i}\right)\right)$ is the horizontal component of the wave wave number, $K=\omega / c_{0}$ is the natural wave number, $M_{i}=M_{1,2}$ the mean Mach in the medium of incidence and $\theta_{i}=\theta_{1,2}$, the angle of incidence. Then, we obtain the PridmoreBrown $^{44}$ equation for $P$ :

$$
P^{\prime \prime}+\frac{2 \alpha M^{\prime}(y)}{K-M(y) \alpha} P^{\prime}+\left[(K-M(y) \alpha)^{2}-\alpha^{2}\right] P=0
$$

where the prime notation stands for a derivation with respect to $y$. The time-averaged energy equation, as given by Myers $^{38}$ is:

$$
\nabla \cdot\langle\boldsymbol{I}\rangle=-\langle\mathcal{D}\rangle
$$

with $\boldsymbol{I}$ the energy flux given by $\boldsymbol{I}=\left(\rho_{0} \boldsymbol{v}+\rho \boldsymbol{v}_{0}\right)\left(c_{0}^{2} \rho / \rho_{0}+\right.$ $\left.\boldsymbol{v}_{0} \cdot \boldsymbol{v}\right)$, and $\mathcal{D}$ the dissipation, given by $\mathcal{D}=\rho_{0} \boldsymbol{v}_{0} \cdot((\nabla \times$ $\boldsymbol{v}) \times \boldsymbol{v})-\rho \boldsymbol{v} \cdot\left(\left(\nabla \times \boldsymbol{v}_{0}\right) \times \boldsymbol{v}_{0}\right)$. In these expressions, $\rho_{0}$ is the mean density, $\rho$ is the density variation which in adiabatic conditions is equal to $p / c_{0}^{2}, \boldsymbol{v}_{0}$ is the mean velocity field and $\boldsymbol{v}$ is the acoustic particle velocity which we can evaluate using linearized Euler equations with flow as:

$$
\boldsymbol{v}=\left\{\begin{array}{l}
V_{x}(y) \mathrm{e}^{-\mathrm{i}(\omega t-\alpha x)} \\
V_{y}(y) \mathrm{e}^{-\mathrm{i}(\omega t-\alpha x)}
\end{array}\right.
$$

with:

$$
\left\{\begin{array}{l}
\rho_{0} c_{0} V_{x}(y)=\left[\frac{\alpha P}{K-M \alpha}-\frac{M^{\prime} P^{\prime}}{(K-M \alpha)^{2}}\right] \\
\rho_{0} c_{0} V_{y}(y)=\frac{-\mathrm{i} P^{\prime}}{K-M \alpha}
\end{array}\right.
$$

Substituting these values in the definition of $\mathcal{D}$, we find:

$$
<\mathcal{D}>=\frac{M M^{\prime}}{2(K-M \alpha)^{3}} \Im\left[\left(P^{\prime \prime}+\frac{2 \alpha M^{\prime}}{K-M \alpha} P^{\prime}+\left[(K-M \alpha)^{2}-\alpha^{2}\right] P\right) P^{\prime *}\right]
$$

Using Eq. $2,<\mathcal{D}>=0$ and Eq. 3 can now be evaluated to:

$$
\left[\frac{\Im\left(P P^{\prime *}\right)}{(K-M(y) \alpha)^{2}}\right]_{y_{1}}^{y_{2}}=0
$$

meaning that the energy flux of plane waves is conserved across the shear layer.

\section{B. Scattering matrix}

In the domains of constant velocity, the pressure is given by:

$$
\left\{\begin{array}{l}
P(y)=A_{1} \mathrm{e}^{\mathrm{i} \beta_{1} y}+B_{1} \mathrm{e}^{-\mathrm{i} \beta_{1} y} \text { for } y<0 \\
P(y)=A_{2} \mathrm{e}^{-\mathrm{i} \beta_{2} y}+B_{2} \mathrm{e}^{\mathrm{i} \beta_{2} y} \text { for } y>\delta
\end{array}\right.
$$

$$
\frac{\beta_{1}}{\left(K-M_{1} \alpha\right)^{2}}\left|A_{1}\right|^{2}+\frac{\beta_{2}}{\left(K-M_{2} \alpha\right)^{2}}\left|A_{2}\right|^{2}=\frac{\beta_{1}}{\left(K-M_{1} \alpha\right)^{2}}\left|B_{1}\right|^{2}+\frac{\beta_{2}}{\left(K-M_{2} \alpha\right)^{2}}\left|B_{2}\right|^{2} .
$$

We define a scattering matrix $\mathrm{S}$ by:

$$
\left[\begin{array}{l}
B_{1} \\
B_{2}
\end{array}\right]=\mathrm{S}\left[\begin{array}{l}
A_{1} \\
A_{2}
\end{array}\right]=\left[\begin{array}{ll}
R_{1} & T_{2} \\
T_{1} & R_{2}
\end{array}\right]\left[\begin{array}{l}
A_{1} \\
A_{2}
\end{array}\right]
$$

where $R_{1}$ (respectively $R_{2}$ ) is the reflection coefficient for a wave coming from medium 1 (resp. 2); $T_{1}$ (respectively $T_{2}$ ) is the transmission coefficient for a wave coming from medium 1 (resp. 2). With the correct normalization on the amplitudes, one can re-write equation 9 as $\tilde{\mathrm{S}}^{\dagger} \tilde{\mathrm{S}}=\mathrm{I}$, where the $\dagger$ exponent stands for Hermitian transposition, $\tilde{S}$ is the normalized scattering matrix and I is the 4-by- where $\beta_{j}^{2}=\left(K-M_{j} \alpha\right)^{2}-\alpha^{2}(j=1,2)$. Using Eq. 7 and the formalism shown in Fig. 1 we obtain:

4 identity matrix. This last relation is exactly the same relation as in the no-flow case. The scattering coefficients then satisfy the four relations:

$$
\left\{\begin{array}{l}
\left|R_{1}\right|^{2}+\frac{\beta_{2}}{\beta_{1}}\left(\frac{K-M_{1} \alpha}{K-M_{2} \alpha}\right)^{2}\left|T_{1}\right|^{2}=1 \\
\left|R_{2}\right|^{2}+\frac{\beta_{1}}{\beta_{2}}\left(\frac{K-M_{2} \alpha}{K-M_{1} \alpha}\right)^{2}\left|T_{2}\right|^{2}=1 \\
\left|R_{1}\right|=\left|R_{2}\right|, \\
\left|T_{1}\right|=\frac{\beta_{1}}{\beta_{2}}\left(\frac{K-M_{2} \alpha}{K-M_{1} \alpha}\right)^{2}\left|T_{2}\right| .
\end{array}\right.
$$

Equations 11 show that the relation between the scattering coefficients is independent of the shear layer thickness. However, their values do depend on it (see appendix 


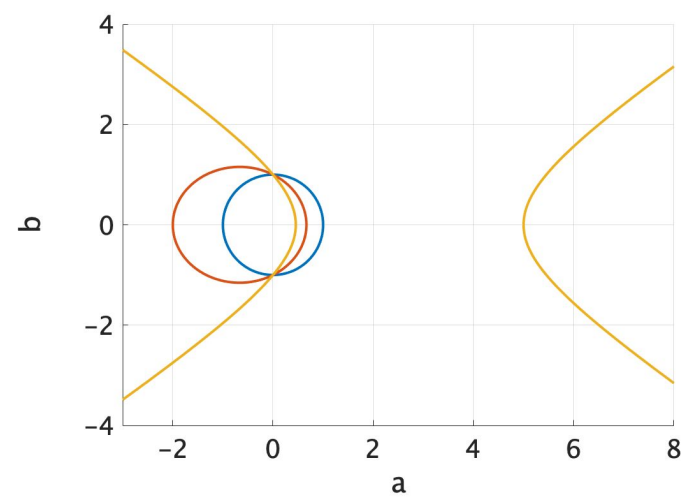

FIG. 2. (color online) Dispersion relation curve in the $(a ; b)$ plane ( $a$ and $b$ being the dimensionless horizontal and vertical wave numbers), when $M_{0}=0$ (blue circle) ; $M_{0}=0.5$ (red ellipse) and $M_{0}=1.2$ (yellow hyperbola).

A). In the case when on of the incident waves has zeroamplitude, Eq. 11 verifies the relation found by Jones ${ }^{26}$.

\section{Dispersion relation and anomalous refraction properties}

A useful representation of scattering between two media of different characteristics is to represent dispersion relations in both media in the $(a, b)$ plane $(a$ and $b$ being respectively the horizontal and vertical component of the wave number, normalized by $K$ ). In our case, we have the following dispersion relation:

$$
a_{j}^{2}+b_{j}^{2}=\left(1-M_{j} a_{j}\right)^{2},
$$

with $j$ the index of the considered medium. In this formulation, we recognize a conic equation, with an eccentricity directly given by $\left|M_{j}\right|$. Therefore, we will have a circle of radius 1 in the $(a, b)$ plane if $M_{j}=0$; an ellipse if $\left|M_{j}\right|<1$; a parabola if $\left|M_{j}\right|=1$; an hyperbola if $\left|M_{j}\right|>1$. This property is illustrated in Fig. 2. Plotting such a relation gives access to both the group and the phase velocity directions for the refracted wave (see Fig. 3). As we study Gaussian beams, the direction of the group velocity, given by the direction of the normal to the ellipse at the point $(a(\theta), b(\theta))$ will have a particular interest as it will give the apparent direction of the beam. The beams are built by summing weighed plane waves as follows:

$$
p(x, y)=\int_{-\infty}^{+\infty} \mathrm{e}^{-\left(\alpha-\alpha_{0}\right)^{2} / w^{2}} p(\alpha, x, y) \mathrm{d} \alpha,
$$

with $\alpha_{0}$ the horizontal wave number in the (imposed) main direction of the beam, and $w$ the waist of the beam. In Fig. 3, these beams are represented for the propagation from a medium 1 at rest to a medium 2 where $M_{2}=0.4$. By looking at the ellipses in the $(a, b)$ plane, and since $\alpha$ is constant between the two media, we can see that for some incidence, $\theta_{1}$ and $\theta_{2}$ can be of opposite sign. This result can be interpreted as an equivalent
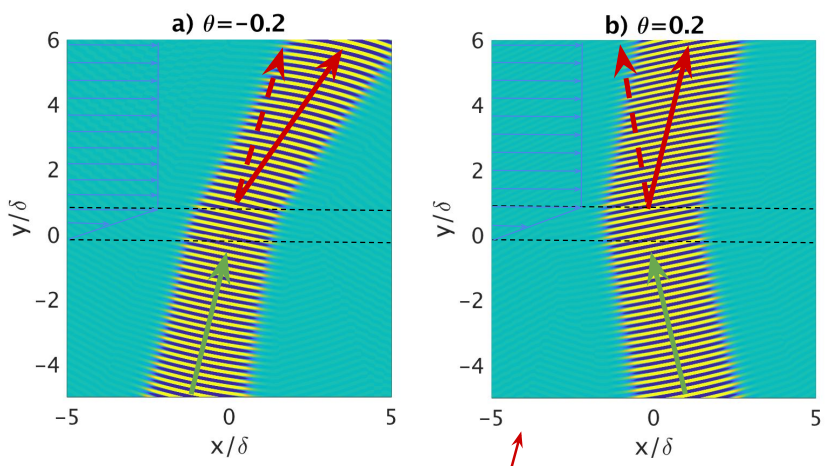

c)

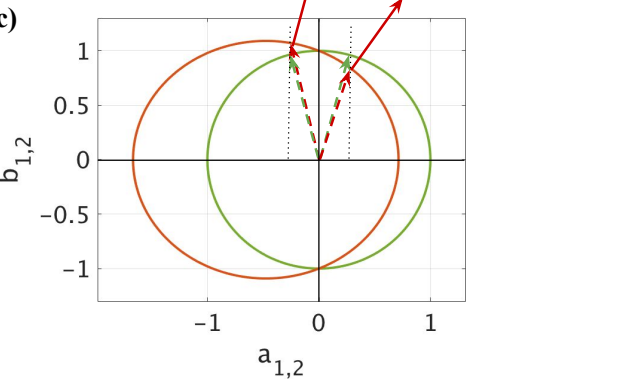

FIG. 3. (color online) Beams coming from medium 1 with an incident angle a) $\theta=-0.2$ or b) $\theta=0.2$. c) represents the dispersion relations in the plane $\left(a_{i}, b_{i}\right)$, with Mach numbers $M_{1}=0$ and $M_{2}=0.4$. Conservation of $a$ at the interfaces allows us to find the direction of the phase velocity by finding the point of the ellipse with abscissa $a$. The normal to the ellipse in this point then gives the direction of the group velocity (i.e. the visible direction of the beam). Green items are linked to medium 1 and red items to medium 2. Solid arrows stand for group velocity direction and dashed arrows for phase velocity. In medium 1 , only one arrow is represented as phase and group velocity are co-linear in a medium at rest.

negative refractive index, analog to what is observed for electromagnetic waves in left handed materials ${ }^{49}$ and in hyperbolic meta-materials ${ }^{9,19,27}$. The shear layer thickness will influence the value of the moduli of $R$ and $T$ but not the direction of the transmitted beam which only depends on the Mach numbers.

\section{REFLECTION ON AN IMPEDANCE WALL}

We now consider an acoustically treated wall with uniform admittance $Y_{0}$ placed in $y=0$ with a shear flow (see Fig. 4). $Y_{0}$ is such that its imaginary part stands for the reactance of the wall, and its real part stands for the resistance of the wall $\left(\Re\left(Y_{0}\right)<0\right.$ means that there are losses at the wall, $\Re\left(Y_{0}\right)>0$ means that there is gain at the wall). 


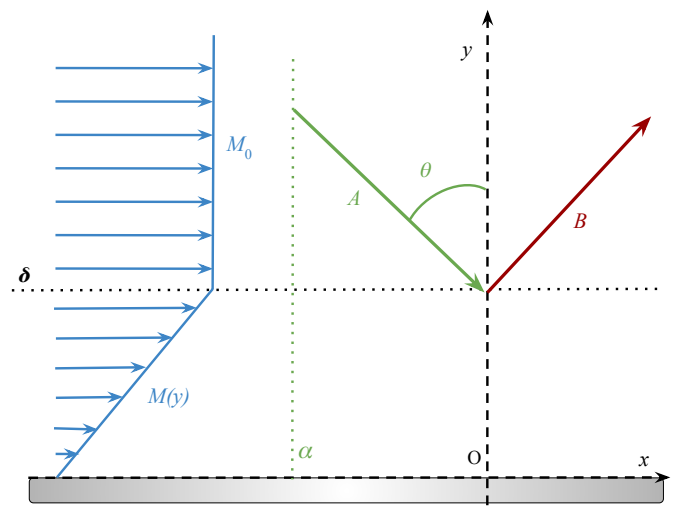

FIG. 4. Geometry of the problem for the reflection of a plane on an admittance wall.

\section{A. Energy conservation}

We know that Eq. 7 is still valid, and that the pressure field for $y>\delta$ is such that:

$$
P(y)=A \mathrm{e}^{-\mathrm{i} \beta y}+B \mathrm{e}^{+\mathrm{i} \beta y},
$$

where $\beta^{2}=\left(K-M_{0} \alpha\right)^{2}-\alpha^{2}$. Now, due to the impedance wall, there is a mixed boundary condition involving the admittance $Y_{0}$ at $y=0$ :

$$
P^{\prime}(0)=\mathrm{i} K Y_{0} P(0)
$$

The scattering problem that we consider is only with reflection and the energy flux conservation 7 applied between $y_{1}=0$ and $y_{2}=\delta$ leads to:

$$
|R|^{2}=1+\frac{\left(\alpha^{2}+\beta^{2}\right)}{K \beta}|P(0)|^{2} \Re(Y) .
$$

As long as the flow is subsonic, one can check that $\beta$ is positive. Therefore, the sign of $|R|^{2}-1$ will only depend on the sign of $\Re(Y)$, whatever the mean flow velocity or the shear layer thickness. In particular, as noted by Campos $^{7}$, if the wall is purely reactive $(\Re(Y)=0)$ then the reflection will always be perfect i.e. $|R|=1$.

\section{B. Non-reciprocal perfect absorption}

The computation of $R$ will be achieved numerically using the method detailed in appendix B. The addition of flow leads to non-reciprocity testified in the value of the absorption coefficient, defined as $A=1-|R|^{2}$. Indeed, an incoming plane wave will not be absorbed to the same extend at the wall depending on whether it comes in the direction of the flow or against the flow. This nonreciprocal property is highlighted in Fig. 5, for a liner of admittance $Y=0.1 i-0.1$. This figure shows clearly that varying $K \delta$ allows to reach high absorption for one incidence. This is a typical behaviour as long as the shear layer thickness is large enough. One can also remark on this figure that the higher the Mach number and the

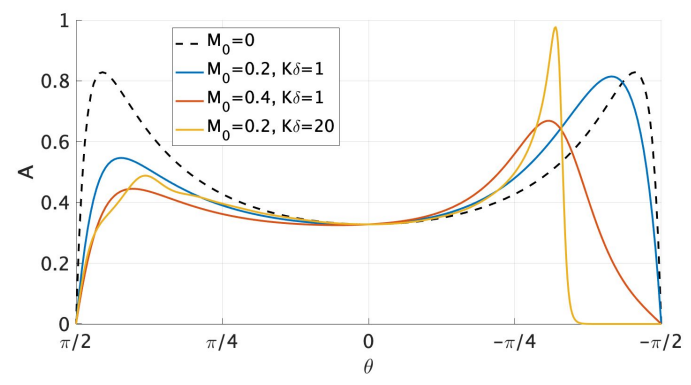

FIG. 5. (color online) Value of the absorption coefficient $A$ as a function of $\theta$ for several flows, described by their mean Mach number $M_{0}$ and the height of their shear layer $\delta$ for a liner of admittance $Y=0.1 i-0.1$.
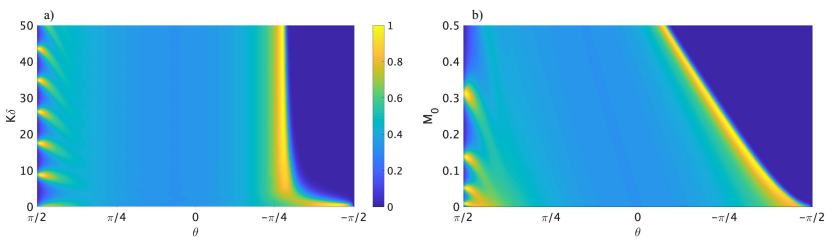

FIG. 6. (color online) Value of the absorption coefficient $A$ for a liner of admittance $Y=0.1 \mathrm{i}-0.1$ as a function of a) $\theta$ and $K \delta$ for $M_{0}=0.1$, b) $\theta$ and $M$ for $K \delta=30$.

height of the shear layer, the more non-reciprocal the system becomes. For a clearer view of this asymmetry, we plot in Fig. 6 the value of the absorption coefficient as a function of both $\theta$ and $K \delta$ and $\theta$ and $M_{0}$. A clear difference can be noted whether incident waves come from the left (in the direction of the flow) or from the right (against the flow). In particular, what is striking on Fig. 6 is that, above a certain thickness of shear layer, waves coming against the flow with a wide angle are fully reflected. This could be explained by a strong refraction of the rays, such that the incident wave does not see the effects of the liner before being pushed back towards the "sky". This corresponds to the "turning points", evoqued by Rienstra ${ }^{48}$. On the other side, waves coming with the flow $(\theta>0)$ with an almost grazing incidence can either be perfectly reflected or perfectly absorbed depending on the shear layer thickness. The quasi-periodic phenomenon which appears is related to resonance phenomena inside the shear layer. For the previous Mach number, for small incidence angles, the absorption does not vary a lot. Therefore, we look at the variation of $A$ with respect to both $\theta$ and $M_{0}$ to see if the peak absorption can be displaced towards normal incidence for higher Mach numbers (see Fig. 6). The position of the near-toperfect absorption for negative $\theta$ then depends linearly on $M_{0}$. Using numerical simulations, we want to design a system which would enhance the absorption of a given liner by adding a specific flow above it, so that it reaches perfect absorption, or zero reflection. The admittance of the liner is fixed at $Y_{0}=-0.1+0.1 \mathrm{i}$, and the incident 

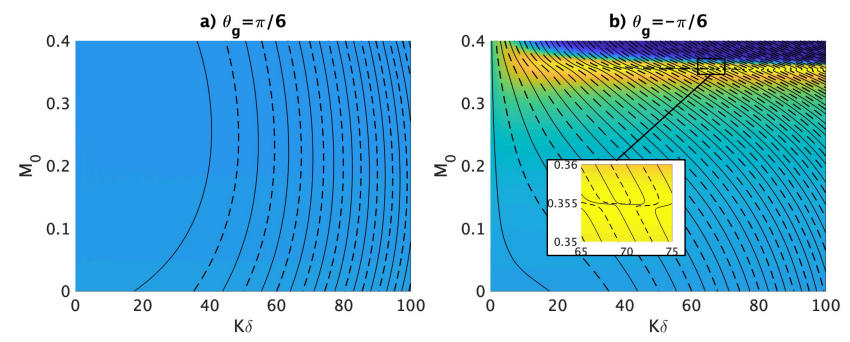

FIG. 7. (color online) Value of $A$ as a function of $M_{0}$ and $K \delta$ for waves coming either in the direction of the flow (case a) or counter-flow (case b). Black lines show the values of $M_{0}$ and $K \delta$ for which $\Re(R)=0$ (dashed lines) or $\Im(R)=0$ (solid lines). It appears that we can find one crossing point, corresponding to $|R|=0$ (i.e. $A=1$ ) for waves coming counter-flow only, for $K \delta=69.68$ and $M_{0}=0.355$.

angle such that $|\theta|=\frac{\pi}{6}$. Without flow, in such a situation $A \approx 0.36$ (see Fig. 8). In order to get a physically consistent simulation, it is not the direction of the phase velocity which is held constant, but the direction of the group velocity $\theta_{g}$. A relation between $\theta$ and $\theta_{g}$ can be found using the dispersion relation (as shown in Fig. 2):

$$
\theta_{g}=\arctan \left[\tan (\theta)+\frac{M_{0}}{\cos (\theta)}\right]
$$

or $\theta=\arcsin \left[\sin \left(\theta_{g}\right) \cos \left(\theta_{g}\right) \sqrt{\frac{1}{\cos ^{2}(\theta)}-M_{0}^{2}}-\right.$ $\left.M_{0} \cos ^{2}\left(\theta_{g}\right)\right]$. We now look at the variations of $|R|$ as both $M_{0}$ and $K \delta$ vary for $\theta_{g}=\pi / 6$. In Fig. 7, an optimum is found, leading to no reflection when $\theta_{g}=-\pi / 6$ (counter-flow propagation). We precisely read it as being linked to the values $M_{0}=0.355$ and $K \delta=69.68$. Non-reciprocity can also be pointed out here, as there is no way to reach perfect absorption if the opposite incidence is considered. We can check that theses values are also associated with an optimal absorption in terms of incidence by plotting $A$ as a function of $\theta$ without flow or with a flow corresponding to the optimum previously identified. Figure 8 gives us this confirmation: the absorption (the absorption coefficient is 0.36 without flow) can be enhanced all the way to perfect absorption with the right flow parameters.

Let us now illustrate the non-reciprocal perfect absorption with Gaussian beams following either upstream or downstream propagation. The results are plotted in Fig. 9. The reflection becomes highly non-reciprocal when flow is added: perfect absorption here is only achieved for waves coming counter-flow. Waves traveling in the direction of the flow actually encounter more reflection than they did in a medium at rest on the same liner (as pointed in Fig. 8). This behaviour is in agreement with the refraction of acoustic rays in shear flow: rays are pushed towards the regions of low local wave speed, consequently they are trapped near the wall when

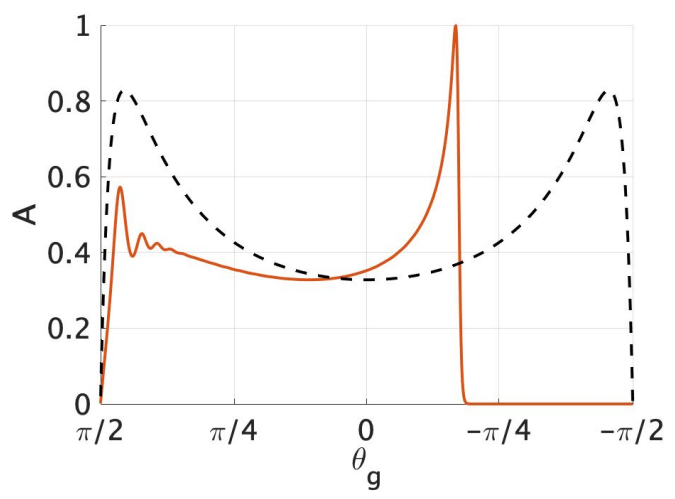

FIG. 8. Value of $A$ as a function of $\theta_{g}$ with and without flow $\left(M_{0}=0.355\right)$, for $Y=-0.1+0.1 \mathrm{i}$ and $K \delta=69.68$.
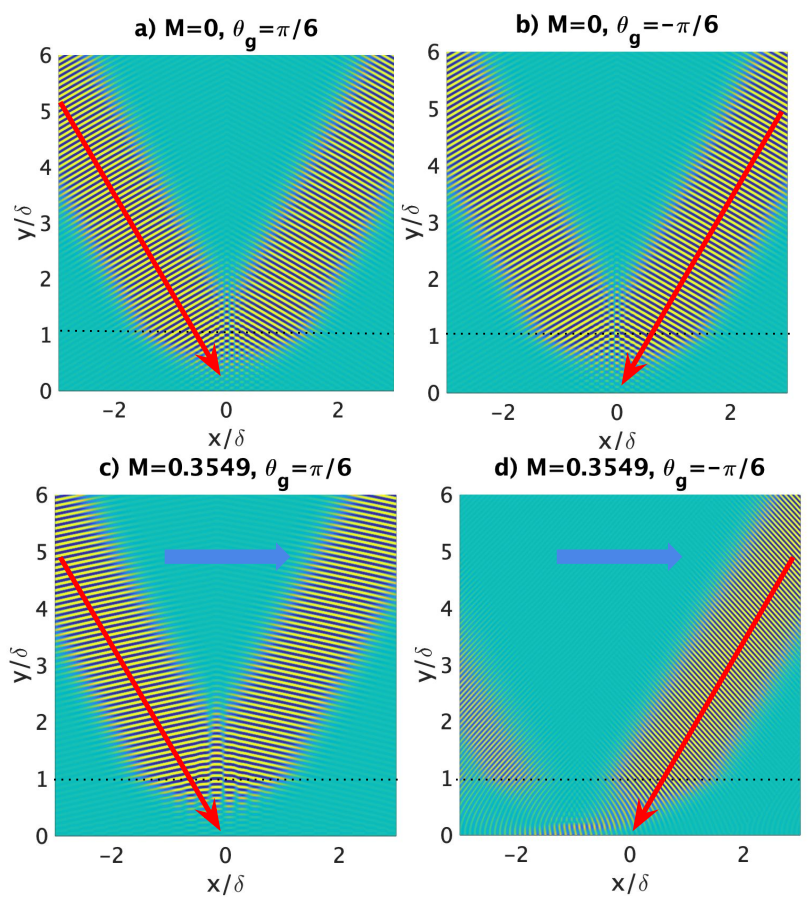

FIG. 9. (color online) Pressure field for an incident Gaussian beam coming with a constant incident angle $\theta_{g}=\frac{\pi}{6}$ between its group velocity and the normal to the wall, either from the left (cases a and c) or from the right (cases b and d) on a liner of admittance $Y_{0}=-0.1+0.1 i$ in a medium at rest (cases $\mathrm{a}$ and $\mathrm{b}$ ) or in which we impose a flow with Mach number $M_{0}=0.355$ and a boundary layer such that $K \delta=69.68$. Red arrows show the direction of the group velocity, blue arrows show the direction of the flow.

propagating upstream and pushed towards the sky when propagating downstream.

\section{Non-reciprocal Goos-Hänchen shift}

When the reflection implies an incidence-depending phase shift, Gaussian beams are shifted along the wall. 


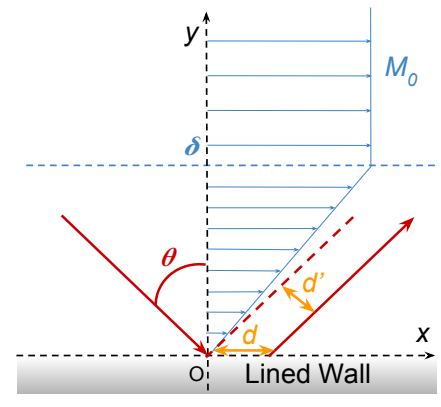

FIG. 10. Scheme of the lateral shift phenomenon for an incident beam. $d$ is the shift along the wall, $d^{\prime}$ is the shift perpendicularly to the direction of the beam.

This phenomenon is called Goos-Hänchen shift ${ }^{22}$, and can be quantified using Artmann's formula ${ }^{2}$ :

$$
d=-\frac{\partial \phi_{R}}{\partial \alpha}=-\frac{(1+M \sin (\theta))^{2}}{K \cos (\theta)} \frac{\partial \phi_{R}}{\partial \theta},
$$

where $\phi_{R}$ is the angle of the reflection coefficient, and $d$ is the lateral shift, as displayed in Fig. 10. We also define the displacement of the beam perpendicularly to its direction of propagation: $d^{\prime}=\cos (\theta) d$.

\section{Without flow}

We know that, without flow, the reflection coefficient is given by:

$$
R=\frac{\cos (\theta)+Y_{0}}{\cos (\theta)-Y_{0}}
$$

In the following, $Y_{0}=Y_{1}+\mathrm{i} Y_{2}$. Then its angle $\phi_{R}$ is given by:

$$
\phi_{R}=\arctan \left(\frac{Y_{2}}{\cos (\theta)+Y_{1}}\right)+\arctan \left(\frac{Y_{2}}{\cos (\theta)-Y_{1}}\right) .
$$

In the case of a lossless wall, $Y_{1}=0$, Eq. 20 reduces to

$$
\phi_{R}=2 \arctan \left(\frac{Y_{2}}{\cos (\theta)}\right) \text {. }
$$

We can then compute the value of $d^{\prime}$, and we get:

$$
K d^{\prime}=-\frac{2 Y_{2} \sin (\theta)}{\cos ^{2}(\theta)+Y_{2}^{2}} .
$$

By differentiating Eq. 22 with respect to $Y_{2}$, we find a maximum displacement of the beam $K d_{\text {max }}^{\prime}= \pm \tan (\theta)$ for $Y_{2}= \pm \cos (\theta)$. Thus, it appears that, for lossless walls, the value of $K d_{\max }^{\prime}$ is small for small incident angles. Nevertheless, it is possible to make this limitation disappear by adding losses to the liner. Indeed, in the case when $Y_{1} \neq 0$, we find that $K d^{\prime}$ can take much larger values. With losses $\left(Y_{1} \neq 0\right)$, Eq. 22 takes the form:

$$
K d^{\prime}=-Y_{2} \sin (\theta) \frac{\cos ^{2}(\theta)+|Y|^{2}}{\left[\left(\cos (\theta)+Y_{1}\right)^{2}+Y_{2}^{2}\right]\left[\left(\cos (\theta)-Y_{1}\right)^{2}+Y_{2}^{2}\right]}
$$

where, for $Y_{1}= \pm \cos (\theta)$ and $Y_{2}$ is close to zero, the denominator can go to zero leading to large $K d^{\prime}$. This result is also the one leading to perfect absorption. Therefore, large lateral shift is associated with large absorption coefficient. This phenomenon is easily understandable if one is to look at the phase portrait of the reflection coefficient when it varies with $\theta$ (see Fig. 11): phase variation of $R$ is enhanced near zero reflection.

\section{With Flow}

When flow is added, the Goos-Hänchen displacement has to be computed numerically. It is then interesting to study the impact of both the flow and the wall admittance on the beam displacement. Results shown in Fig. 12 illustrate that the effect of the flow is predominant. Indeed, the two maximal values of displacement visible without flow for $\theta \approx \pm \pi / 6$ are still visible when the flow is added but are negligible compared to the effect of the flow with a much larger displacement at $\theta \approx-\pi / 4$. Remark that this displacement is comparable to the large displacement of beams observed in the case of reflection on multi-layered materials in optics ${ }^{11,15,50}$.
As in the previous section, where we illustrated nonreciprocal perfect absorption, Gaussian beams can nicely illustrate the Goos-Hänchen effect (Fig. 13). By choosing the angle for which the flow has the most visible effect $(\theta=-0.89)$, we see a large lateral shift. Besides, a strong non-reciprocal effect is demonstrated by taking the opposite angle.

\section{CONCLUSION}

In the global context of acoustics with flow we have shown that the presence of a shear layer leads to nonreciprocal effects whose origin can be found in the known effects due to convection. We have illustrated some of these phenomena by considering diffraction by a shear layer that can lead to negative refraction, as well as absorption by an acoustic material and lateral displacement of a beam along this material which both depend on the direction of propagation relatively to the flow. In this paper, we have only considered homogeneous problems along the direction of flow. Interesting new effects appear as soon as inhomogeneities are considered along the flow direction. 

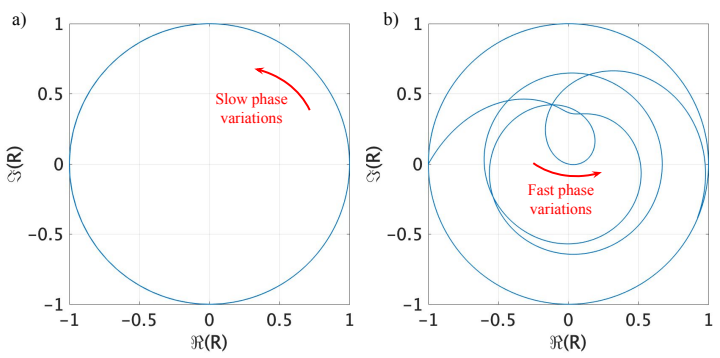

FIG. 11. Phase portrait of $R$ as $\theta$ varies from $-\pi / 2$ to $+\pi / 2$, with $M_{0}=0.23, K \delta=15.04$ and a) $Y_{0}=0.1 i$, b) $Y_{0}=0.1 i-0.2$. When losses are added on the wall and in a situation where perfect absorption can be achieved, the reflection coefficient can cross the zero point, and therefore experience a sudden phase jump of amplitude $\pi$, resulting in a high phase gradient and therefore a large lateral displacement of the beam.

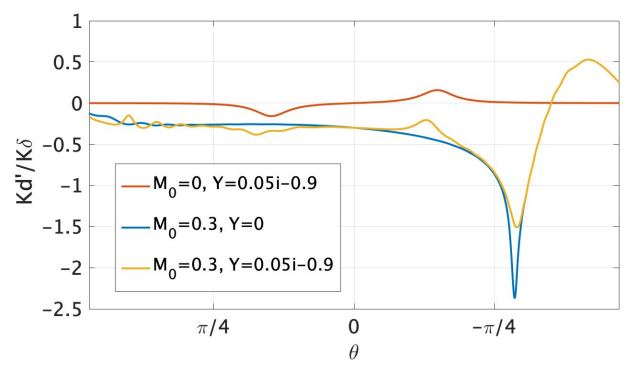

FIG. 12. Value of $d^{\prime} / \delta$ as a function of $\theta_{g}$ for a reflection either on a hard wall or with an acoustic treatment, with and without flow. The flow used here is such that $M_{0}=0.3$ and $K \delta=50$.
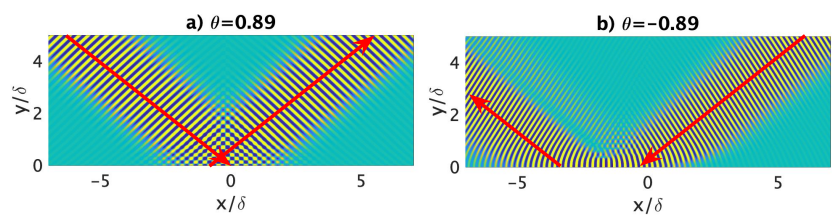

FIG. 13. (color online) Pressure field for Gaussian beam with a constant main direction $\theta_{g}= \pm 0.877$, reflecting on a hard wall, with $M_{0}=0.3$ and $K \delta=30$. In case a), the beam comes in the direction of the flow and is slightly displaced backwards in case b), the beam comes against the direction of the flow and is widely displaced forwards. The black dashed line shows the height of the shear layer.

\section{APPENDIX A: NUMERICAL DETERMINATION OF THE SCATTERING COEFFICIENTS IN A TRANSMISSION PROBLEM}

To find the scattering coefficients, we need to solve Eq. 2. For this, we define $Q$ :

$$
Q(y)=\frac{P^{\prime}(y)}{K P(y)} .
$$

Then Eq. 2 can be re-written as a first-order differential equation for $Q$, and becomes a Ricatti equation. Moreover, to make its integration easier, a dimensionless variable $s$ is introduced, such that $s=\frac{y}{\delta}$. The integration will then be done on $[0,1]$. Finally,the equation to solve is:

$Q^{\prime}(s)=-K \delta\left[Q(s)^{2}+\left(1-M_{0} a s\right)^{2}-a^{2}\right]-\frac{2 M_{0} a}{1-M_{0} a s} Q(s)$,

where $M_{0}=M_{2}-M_{1}$ and $a=\frac{\alpha}{K}$. Solving for the whole scattering matrix requires the study of two cases:

- if the incident wave comes from medium $1, A_{2}$ is null, and Eq. 8 gives us the following boundary condition in $s=1$ :

$$
Q(1)=\mathrm{i} b_{2},
$$

where $b_{2}=\beta_{2} / K$. Equation A2 could have be solved with a common numerical method (such as a Runge-Kutta algorithm), but large heights of shear layer resulted in divergences in the result. Thus, we implemented a Magnus-Moebius scheme (as described $\operatorname{in}^{43}$ ) for $s$ going from 1 to 0 . In order to do so, it is necessary to go back to Eq. 2 which, when written on $s$, becomes:

$P^{\prime \prime}+\frac{2 a\left(M_{2}-M_{1}\right)}{1-\left[M 1+\left(M_{2}-M_{1}\right) s\right] a} P^{\prime}+(K \delta)^{2}\left[\left(\left(1-M_{1}+\left(M_{2}-M_{1}\right) s\right) a\right)^{2}-\right.$

When put under a matrix form, Eq. A4 becomes: 


$$
\mathrm{d}_{s}\left(\begin{array}{c}
P \\
P^{\prime}
\end{array}\right)=\left[\begin{array}{cc}
0 & 1 \\
-(K \delta)^{2}\left[\left(\left(1-M_{1}+\left(M_{2}-M_{1}\right) s\right) a\right)^{2}-a^{2}\right] & -\frac{2 a\left(M_{2}-M_{1}\right)}{1-\left[M 1+\left(M_{2}-M_{1}\right) s\right] a}
\end{array}\right]\left(\begin{array}{c}
P \\
P^{\prime}
\end{array}\right) .
$$

The matrix involved in Eq. A5 will later be denoted $\mathrm{M}(s)$ Discretizing the domain in $N+1$ points (intervals of size $h=1 / N$ ) allows us to apply the following scheme :

$$
\left(\begin{array}{c}
P(n h) \\
P^{\prime}(n h)
\end{array}\right)=\mathrm{e}^{\mathrm{H}_{n}}\left(\begin{array}{c}
P((n+1) h) \\
P^{\prime}((n+1) h)
\end{array}\right)
$$

where $\mathbf{H}_{n}=-h \mathrm{M}[(n+1 / 2) h]$. Note that the exponential in Eq. A6 is a matrix exponential. At each iteration, it is possible to compute $Q(n h)$ from $Q((n+1) h)$ :

$$
Q(n h)=\frac{\frac{E_{2,1}}{K \delta}+E_{2,2} Q((n+1) h)}{E_{1,1}+K \delta E_{1,2} Q((n+1) h)}
$$

where the $E_{i, j}$ are the coefficients of $\mathrm{e}^{\mathrm{H}_{n}}$. After $N$ iterations, we can access $R_{1}$ via Eq. 8, and we find:

$$
R_{1}=\frac{\mathrm{i} b_{1}-Q(0)}{\mathrm{i} b_{1}+Q(0)}
$$

where $b_{1}=\frac{\beta_{1}}{K}$.

- if the incident wave comes from medium $2, A_{1}$ is null and we can now write a boundary condition in $s=0$ :

$$
Q(0)=-\mathrm{i} b_{1} \text {. }
$$

Once again, Eq. A5 is solved iteratively for $s$ going from 0 to 1 . This time, we have:

$$
\left(\begin{array}{c}
P((n+1) h) \\
P^{\prime}((n+1) h)
\end{array}\right)=\mathrm{e}^{-\mathrm{H}_{n}}\left(\begin{array}{c}
P(n h) \\
P^{\prime}(n h)
\end{array}\right)
$$

Thus:

$$
Q((n+1) h)=\frac{\frac{E_{2,1}}{K \delta}+E_{2,2} Q(n h)}{E_{1,1}+K \delta E_{1,2} Q(n h)},
$$

and we can access $R_{2}$ using:

$$
R_{2}=\frac{\mathrm{i} b_{2}+Q(1)}{\mathrm{i} b_{2}-Q(1)} e^{-2 \mathrm{i} K \delta b_{2}} .
$$

The determination of transmission coefficients $T_{1}$ and $T_{2}$, now that $Q$ is known in $[0,1]$, requires to integrate Eq. A4 one more time, but in the opposite direction. Here also, we will need to study the two cases of upward and downward traveling incident waves.

- if the incident wave comes from medium 1 , we know from Eq. 8 that the boundary condition in $s=0$ is:

$$
\tilde{P}(0)=1+R_{1},
$$

where $\tilde{P}$ is the $y$-dependant component of the pressure field normalized by the incident amplitude $A_{1}$. Then, by solving for $\tilde{P}$ on $[0,1]$ using the scheme in Eq. A10, we can access $T_{1}$ using:

$$
T_{1}=\tilde{P}(1) \mathrm{e}^{-\mathrm{i} K \delta b_{2}} .
$$

- if the incident wave comes from medium 2, then this time the boundary condition is:

$$
\tilde{P}(1)=\mathrm{e}^{-\mathrm{i} K \delta b_{2}}+R_{2} \mathrm{e}^{\mathrm{i} K \delta b_{2}},
$$

where $\tilde{P}(s)$ is normalized by $A_{2}$. Then, by solving for $\tilde{P}$ on $[1,0]$ using the scheme in Eq. A6, we can access $T_{2}$ using:

$$
T_{2}=\tilde{P}(0)
$$

The whole matrix can then be reconstituted. It turns out from Eq. A2 that three parameters will influence the values of the scattering coefficients: the dimensionless width of the shear layer $K \delta$, the relative Mach number $M_{2}-M_{1}$ and the direction of the incident wave given by the horizontal wave number $a$.

\section{APPENDIX B: NUMERICAL DETERMINATION OF $R$ IN A REFLECTION PROBLEM}

In order to determine $R$, we apply the same method as for the transmission problem : solving Eq. A2 for $s$ in $[0 ; 1]$, but this time the boundary condition will be given by the wall admittance:

$$
Q(0)=\mathrm{i} Y_{0}
$$

Here also, the resolution is led to its end using a MagnusMoebius scheme ${ }^{30}$ of the second order as described in ${ }^{43}$. In order to compute the solution, we go back to Eq. A5, and after writing $P$ and $P^{\prime}$ in discrete forms, we get:

$$
\left(\begin{array}{c}
P((n+1) h) \\
P^{\prime}((n+1) h)
\end{array}\right)=\mathrm{e}^{\mathrm{H}_{n}}\left(\begin{array}{c}
P(n h) \\
P^{\prime}(n h)
\end{array}\right)=\left[\begin{array}{cc}
E_{1,1} & E_{1,2} \\
E_{2,1} & E_{2,2}
\end{array}\right]\left(\begin{array}{c}
P(n h) \\
P^{\prime}(n h)
\end{array}\right)
$$


where $\mathrm{H}_{n}=h \mathrm{M}[(n+1 / 2) h]$. Thus:

$$
Q((n+1) h)=\frac{\frac{E_{2,1}}{K \delta}+E_{2,2} Q(n h)}{E_{1,1}+K \delta E_{1,2} Q(n h)} .
$$

$R$ is finally given by:

$$
R=\frac{\mathrm{i} b+Q(1)}{\mathrm{i} b-Q(1)} \mathrm{e}^{-2 \mathrm{i} b K \delta} .
$$

${ }^{1}$ Amiet, R. K. (1978). "Refraction of sound by a shear layer," Journal of Sound and Vibration 58, 467-482.

${ }^{2}$ Artmann, K. (1947). "Berechnung der Seitenversetzung des totalreflektierten Strahles (Calculation of the lateral displacement of the totally reflected beam)," Annalen der Physik 333.

${ }^{3}$ Brambley, E. J. (2009). "Fundamental problems with the model of uniform flow over acoustic linings," Journal of Sound and Vibration 322(4-5), 1026-1037, doi: 10.1016/j.jsv.2008.11.021.

${ }^{4}$ Brambley, E. J. (2011). "A well-posed boundary condition for acoustic liners in straight ducts with flow," AIAA Journal 7-9.

${ }^{5}$ Brand, R. S., and Nagel, R. T. (1982). "Reflection of sound by a boundary layer," Journal of Sound and Vibration 85, 31-38.

${ }^{6}$ Breazeale, M. A., Adler, L., and Scott, G. W. (1977). "Interaction of ultrasonic waves incident at the Rayleigh angle onto a liquidsolid interface," Journal of Applied Physics 530, doi: 10.1063/1.323677

${ }^{7}$ Campos, L. M. B. C., and Kobayashi, M. H. (2013). "On an acoustic oscillation energy for shear flows," International Journal of Aeroacoustics 12(1+2), doi: https://doi.org/10.1260/ 1475-472X.12.1-2.123.

${ }^{8}$ Cantrell, R. H., and Hart, R. W. (1964). "Interaction between sound and flow in acoustic cavities : mass, momentum and energy considerations," Journal of the Acoustical Society of America 697.

${ }^{9}$ Christensen, J., and de Abajo, F. J. G. (2012). "Anisotropic metamaterials for full control of Acoustic Waves," Physical Review Letters 108(12), 124301, https://link.aps.org/doi/ 10.1103/PhysRevLett.108.124301, doi: 10.1103/PhysRevLett. 108.124301.

${ }^{10}$ Declercq, N. F., Degrieck, J., Briers, R., and Leroy, O. (2004). "Theory of the backward beam displacement on periodically corrugated surfaces and its relation to leaky Scholte-Stoneley waves," Journal of Applied Physics 96(11), 6869-6877, doi: $10.1063 / 1.1808247$.

${ }^{11}$ Declercq, N. F., Van des Abeele, F., Degrieck, J., and Leroy, O. (2004). "The Schoch effect to distinguish between different liquids in closed containers," IEEE Transactions on Ultrsonics, Ferroelectrics and Frequency Control 51(10), 1354-1357.

${ }^{12}$ Delaroche, F. (1816) "Sur l'influence que le vent exerce dans la propagation du son, sous le rapport de son intensité," Ann. Chim $\mathbf{1}(176)$.

${ }^{13}$ Emile, O., Galstyan, T., Le Floch, A., and Bretenaker, F. (1995). "Measurement of the nonlinear Goos-Hänchen effect for Gaussian optical beams," Physical Review Letters 75(8), 1511-1513, doi: 10.1103/PhysRevLett.75.1511.

${ }^{14} \mathrm{Fa}$, L., Xue, L., Fa, Y., Han, Y., Zhang, Y., Cheng, H., Ding, P., Li, G., Tan, S., Bai, C., Xi, B., Zhang, X., and Zhao, M. (2017). "The acoustic Goos-Hänchen effect," Science China - Physics, Mechanics \& Astronomy 60, doi: 10.1007/s11433-017-9052-9.

${ }^{15}$ Farmani, A. (2018). "Graphene Plasmonic : Switching Applications," in Graphene- Physics, Chemistry and Biology (WILEYScrivener Publishing LLC), pp. 1-36.

${ }^{16}$ Fleury, R., Sounas, D.L., Sieck, C.F., Haberman, M.R. and Alù, A.(2014). "Sound isolation and giant linear nonreciprocity in a compact acoustic circulator," Science 343(6170), 516-519.

${ }^{17}$ Fleury, R., Sounas, D.L., Haberman, M.R. and Alù, A.(2015). "Nonreciprocal acoustics," Acoustics Today 1(EPFL-ARTICLE223074), 14-21.
${ }^{18}$ Gabard, G. (2013). "A comparison of impedance boundary conditions for flow acoustics," Journal of Sound and Vibration 332(4), 714-724, doi: 10.1016/j.jsv.2012.10.014.

${ }^{19}$ Garcìa-Chocano, V. M., Christensen, J., and Sanchez-Dehesa, J. (2014). "Negative refraction and energy funneling by hyperbolic materials: An experimental demonstration in acoustics," Physical Review Letters 112(14), 11-15, doi: 10.1103/PhysRevLett. 112.144301.

${ }^{20}$ Godin, O. (1997). "Reciprocity and energy theorems for waves in a compressible inhomogeneous moving fluid," Wave Motion 25(2), 143-167, http://www. sciencedirect. com/science/article/pii/S0165212596000376, doi: 10.1016/ S0165-2125 (96) 00037-6.

${ }^{21}$ Goldstein, M., and Rice, E. (1973). "Effect of shear on duct wall impedance," Journal of Sound and Vibration 30(1), 79-84, doi: 10.1016/S0022-460X (73) 80051-3.

${ }^{22}$ Goos, F., and Hänchen, H. (1947). "Ein neuer und fundamentaler Versuch zur Totalreflexion (a new and fundamental attempt at total reflection)," Annalen der Physik 436(7-8), 333-346, doi: 10.1002/andp. 19474360704.

${ }^{23}$ Graham, E. W., and Graham, B. B. (1969). "Effect of a sheared layer on plane waves in a fluid," Journal of the Acoustical Society of America 46(1969), 169-175, doi: 10.1121/1.1911666.

${ }^{24}$ Herbison, S. W. (2011). "Ultrasonic diffraction effects on periodic surfaces," Ph.D. thesis, Georgia Institute of Technology.

${ }^{25}$ Ingard, U. (1959). "Influence of fluid motion past a plane boundary on sound reflection, absorption, and transmission," The Journal of the Acoustical Society of America 31(7), 1035, http://scitation.aip.org/content/asa/journal/jasa/ 31/7/10.1121/1.1907805, doi: 10.1121/1.1907805.

${ }^{26}$ Jones, D.S. (1977). "The Scattering of Sound by a Simple Shear Layer," hilosophical Transactions of the Royal Society of London. Series A, Mathematical and Physical Sciences 1323(284), 287328

${ }^{27} \mathrm{Ju}$, F., Tian, Y., Cheng, Y., and Liu, X. (2018). "Asymmetric acoustic transmission with a lossy gradient-index metasurface," Applied Physics Letters 113(12), 121901, http:// aip.scitation.org/doi/10.1063/1.5032263, doi: 10.1063/1. 5032263.

${ }^{28}$ Koutsoyannis, S. P., Karamcheti, K., and Galant, D. C. (1979). "Acoustic resonances and sound scattering by a shear layer," NASA .

${ }^{29}$ Liu, F., Xianjun, M., Jiaqi, X., Anling, W., and Changchun, Y. (2012). "The Goos-Hänchen shift of wide-angle seismic reflection wave," Science China - Earth Sciences 55(5), 852-857, doi: 10. 1007/s11430-011-4344-5.

${ }^{30} \mathrm{Lu}$, Y. Y. (2005). "A fourth-order Magnus scheme for Helmholtz equation," Journal of Computational and Applied Mathematics 173(2), 247-258, doi: 10.1016/j.cam.2004.03.010.

${ }^{31}$ Mariano, S. (1971). "Effect of wall shear layers on the sound attenuation in acoustically lined rectangular ducts," Journal of Sound and Vibration 19(3), 261-275.

${ }^{32}$ Maznev, A.A., Every, A.G. and Wright, O.B. (2013). "Reciprocity in reflection and transmission: What is a "phonon diode'?," Wave Motion 50(4), 776-784.

${ }^{33}$ Miles, J. W. (1957). "On the reflection of sound at an interface of relative motion," The Journal of the Acoustical Society of America 29(2), 226-228, http://asa.scitation.org/doi/10. 1121/1.1908836, doi: 10.1121/1.1908836.

${ }^{34}$ Miles, J. W. (1958). "On the disturbed motion of a plane vortex sheet," Journal of Fluid Mechanics 4(5), 538-552, doi: 10.1017/ S0022112058000653.

${ }^{35}$ Morfey, C. L. (1971). "Acoustic energy in non-uniform flows," Journal of Sound and Vibration 14(2), 159-170, doi: 10.1016/ 0022-460X (71) 90381-6.

${ }^{36}$ Mungur, P., and Gladwell, G. (1969). "Acoustic wave propagation in a sheared fluid contained in a duct," Journal of Sound and Vibration 9(1), 28-48, doi: 10.1016/0022-460X (69) 90260-0. 
${ }^{37}$ Myers, M. K. (1980). "On the acoustic boundary condition in the presence of flow," Journal of Sound and Vibration 71, 429434, doi: 10.1016/0022-460X (80) 90424-1.

${ }^{38}$ Myers, M. K. (1986). "An exact energy corollary for homentropic flow," Journal of Sound and Vibration 109(2), 277-284, doi: 10. 1016/S0022-460X (86)80008-6.

${ }^{39}$ Naghdi, M. and Farzbod, F.(2018). "Acoustic nonreciprocity in Coriolis mean flow systems," The Journal of the Acoustical Society of America 143(1), 230-236.

${ }^{40}$ Nagel, R. T., and Brand, R. S. (1982). "Boundary layer effects on sound in a circular duct," Journal of Sound and Vibration 85, 19-29.

${ }^{41}$ Nayfeh, A. H. (1973). "Effect of the acoustic boundary layer on the wave propagation in ducts," The Journal of the Acoustical Society of America 54(6), 1737-1742, http://link.aip. org/link/JASMAN/v94/i2/p1076/s1\{\&\}Agg=doi, doi: 10.1121/ 1.1914472 .

${ }^{42}$ Ostashev, Vladimir E., and Wilson, D. Keith (2015) "Acoustics in Moving Inhomogeneous Media, Second Edition," CRC Press.

${ }^{43}$ Pagneux, V. (2010). "Multimodal admittance method in waveguides and singularity behavior at high frequencies," Journal of Computational and Applied Mathematics 234(6), 18341841, http://dx.doi.org/10.1016/j.cam.2009.08.034, doi: 10.1016/j.cam.2009.08.034.

${ }^{44}$ Pridmore-Brown, D. C. (1958). "Sound propagation in a fluid flowing through an attenuating duct," Journal of Fluid Mechanics 4(4), 393-406, doi: 10.1017/S0022112058000537.
${ }^{45}$ Rayleigh (1877). Theory of Sound, Volume II, dover publ ed. (Dover Publications), Chap. XIV, 132-133.

${ }^{46}$ Renou, Y., and Aurégan, Y. (2011). "Failure of the IngardMyers boundary condition for a lined duct: An experimental investigation," The Journal of the Acoustical Society of America 130(1), 52-60, http://asa.scitation.org/doi/10.1121/1. 3586789, doi: 10.1121/1.3586789.

${ }^{47}$ Ribner, H. S. (1957). "Reflection, transmission, and amplification of sound by a moving medium," The Journal of the Acoustical Society of America 29(4), 435, doi: 10.1121/1.1908918.

${ }^{48}$ Rienstra, S. W. (2019). "Solutions and Properties of the Pridmore-Brown Equation," 25th AIAA/CEAS Aeroacoustics Conference 20-24,

${ }^{49}$ Smith, D. R., and Kroll, N. (2000). "Negative refractive index in left-handed materials," Physical Review Letters 85(14), 29332936, doi: https://doi.org/10.1103/PhysRevLett.85.2933.

${ }^{50}$ Tamir, T., and Bertoni, H.L. (1971). "Lateral displacement of optical beams at multilayered and periodic structures," Journal of the Optical Society of America 61(10), 1397-1413, http:// www.opticsinfobase.org/abstract.cfm?URI=josa-61-10-1397, doi: $10.1364 / J O S A .61 .001397$.

${ }^{51}$ Wang, A., and Liu, F. (2014). "The lateral shift of refraction Shwave on an interface of two media," Applied Mechanics and Materials 551, 580-582, doi: 10.4028/www.scientific.net/AMM. 551.580 .

${ }^{52}$ Yeh, C. (1968). "A Further Note on the Reflection and Transmission of Sound Waves by a Moving Fluid Layer," Journal of the Acoustical Society of America 1454(43), 1-3. 